PROCEEDINGS OF THE

AMERICAN MATHEMATICAL SOCIETY

Volume 132, Number 6, Pages 1785-1786

S 0002-9939(03)07228-9

Article electronically published on December 1, 2003

\title{
A SYMMETRY COMPLETENESS CRITERION FOR SECOND-ORDER DIFFERENTIAL EQUATIONS
}

\author{
LARRY BATES \\ (Communicated by Carmen C. Chicone)
}

\begin{abstract}
A simple criterion for the completeness of an infinitesimal automorphism of a second-order differential equation is given.
\end{abstract}

Given a second-order differential equation $Y$ on a connected manifold $M$, and a vector field $X$ on $M$, we say that $X$ is an infinitesimal automorphism of $Y$ if $[\bar{X}, Y]=0$, where $\bar{X}$ is the natural lift of $X$ to $T M$. It is of interest to know when $X$ has a complete flow, which is to say that $X$ is the infinitesimal generator of a oneparameter group of diffeomorphisms of $M$, and hence generates a one-parameter group of automorphisms of $Y$.

Example 1. Consider the second-order equation $Y$ on $M=\mathbb{R}$ with

$$
Y=v \partial_{x}+(1+2 x)\left(1+x^{2}\right)^{-1} v^{2} \partial_{v} .
$$

Then $X=\left(1+x^{2}\right) \partial_{x}$ satisfies $[\bar{X}, Y]=0$, but $X$ is not complete.

In this example, the flow of $Y$ is not complete. However, $Y$ can be incomplete with $X$ complete as in

Example 2. Let $g$ be any left-invariant Lorentz metric on the affine group $G=$ $A(1, \mathbb{R})$. Then the geodesic flow is incomplete, but each left-invariant vector field $X$ on $G$ generates a one-parameter group of symmetries of the geodesic vector field.

In light of these examples, it is perhaps somewhat surprising that the following theorem holds.

Theorem. Let $Y$ be a second-order differential equation on a manifold $M$ with a complete flow $\psi_{t}$. Let $X$ be an infinitesimal automorphism of $Y$. Then $X$ is necessarily complete; that is, it generates a one-parameter group of diffeomorphisms of $M$.

Proof. First observe that in order to prove that $X$ is complete, it suffices to prove the uniform time $\tau$ existence for the local flow. Let $\pi: T M \rightarrow M$ be the projection, and let $\phi_{t}(x),|t|<\tau$ be the local flow of $X$ about $x \in M$. Let $\bar{\phi}_{t}(p)$ be the lifted local flow about $p \in T M$ with $\pi(p)=x$. Then $\phi_{t} \circ \pi=\pi \circ \bar{\phi}_{t}$. Since $[\bar{X}, Y]=0$, $\psi_{t_{*}} \bar{X}=\bar{X}$ for all $t$. This says that if $\psi_{s}(p)=q$ for some $s$, then $\bar{\phi}_{t}(q)=\psi_{s} \circ \bar{\phi}_{t}(p)$.

Received by the editors February 12, 2003 and, in revised form, February 17, 2003.

2000 Mathematics Subject Classification. Primary 34C14.

Key words and phrases. Second-order differential equations, symmetry, completeness.

This research was partially supported by NSERC. 
Hence we may conclude existence of both a $\tau>0$ and a local flow of $\bar{\phi}_{t}(q)$ for all time $t$ with $|t|<\tau$, and thus that of the local flow of $\phi_{t}(\pi(q))$ for the same time $|t|<\tau$ as well.

It follows that we have time $\tau$ existence of the local flow of $\phi_{t}$ for all points $x^{\prime} \in M$ such that there is an integral curve of $Y$ that projects to a curve connecting $x$ and $x^{\prime}$. Continuing in this manner we may also conclude the same time $\tau$ existence for all points $x^{\prime \prime}$ that may be so connected to $x^{\prime}$.

We will be done if we can show that any point $y \in M$ may be connected to $x$ by a finite chain of such points $x, x^{\prime}, x^{\prime \prime}, \ldots, y$, all with the same time $\tau$ existence. For this, it suffices to show that each point $z \in M$ is connected to every other point in some open neighbourhood of $z$. This suffices because a connected manifold is path connected, and so we can find a smooth curve $\gamma:[0,1] \rightarrow M$ such that $\gamma(0)=x$ and $\gamma(1)=y$. Compactness then allows us to select a finite number of points $x, x^{\prime}, x^{\prime \prime}, \ldots, y$ on the image of $\gamma$ from the open covering provided by the following lemma to make the connection. This provides the uniform time $\tau$ estimate that we require.

Lemma. Let $Y$ be a second-order differential equation on a manifold $M$. Then there is an open neighbourhood of $x \in M$ in which every point $x^{\prime}$ is connected to $x$ by the projection $\pi$ of an integral curve of $Y$.

In other words, for all points $x^{\prime}$ in some open neighbourhood of $x$, we may choose an initial velocity $v_{x}$ at $x$ so that the projection of the integral curve of $Y$ through $v_{x}$ contains $x^{\prime}$. The proof of the lemma follows easily from the fact that the exponential map is onto.

This proof is patterned after the discussion of completeness for first-order $G$ structures in [1] and [2]. One should also note that in [3] an infinitesimal automorphism is defined to be complete in order to have a pretty statement about the maximal dimension of the symmetry group.

\section{REFERENCES}

[1] S. Kobayashi, Espaces à connexion de Cartan complets, Proc. Japan Acad. Sci. 30 (1954), 709-710. MR 16:1053d

[2] S. Kobayashi, Transformation groups in differential geometry, volume 70 of Ergebnisse der Mathematik und ihrer Grenzgebiete, Springer-Verlag, New York, 1972. MR 50:8360

[3] O. Loos, Automorphism groups of second-order differential equations, Monatshefte für Mathematik 96 (1983), 195-207. MR 85d:58075

Department of Mathematics, University of Calgary, Calgary, Alberta, Canada T2N $1 \mathrm{~N} 4$

E-mail address: bates@math.ucalgary.ca 